Relations industrielles

Industrial Relations

\title{
Pierre R. TURCOTTE : La QVT une voie vers l'excellence. Montréal, Les Éditions Agence d'Arc, 1988, 276 pp., ISBN 289022-134-2 et ISBN 2-7624-0066-X
}

\section{Roger Zaoré}

\section{Volume 44, numéro 4, 1989}

URI : https://id.erudit.org/iderudit/050550ar

DOI : https://doi.org/10.7202/050550ar

Aller au sommaire du numéro

Éditeur(s)

Département des relations industrielles de l'Université Laval

ISSN

0034-379X (imprimé)

1703-8138 (numérique)

Découvrir la revue

Citer ce compte rendu

Zaoré, R. (1989). Compte rendu de [Pierre R. TURCOTTE : La QVT une voie vers l'excellence. Montréal, Les Éditions Agence d'Arc, 1988, 276 pp., ISBN 289022-134-2 et ISBN 2-7624-0066-X]. Relations industrielles / Industrial Relations, 44(4), 962-963. https://doi.org/10.7202/050550ar

Tous droits réservés @ Département des relations industrielles de l'Université Laval, 1989
Ce document est protégé par la loi sur le droit d'auteur. L’utilisation des services d’Érudit (y compris la reproduction) est assujettie à sa politique d'utilisation que vous pouvez consulter en ligne.

https://apropos.erudit.org/fr/usagers/politique-dutilisation/ 
People who feel competent (self-efficacy) are able to handle stress much better. People differ much in their proneness to stress-related health risks. It is worth to mention here that there are various types of personalities distinguished according to the empirically verified dimensions. For example, introversion-extraversion, neuroticism-stability, psychotism (Eysenck). Cattell has distinguished the following traits: reserved/outgoing, more or less intelligent, humble/assertive, emotional/stable, sober/happy go lucky, expedient/conscientious, shy/venturesome, tough minded/tender minded, trusting/suspicious, practical/imaginative, forthright/shrewd, self assured/apprehensive, conservative/experimenting, group-dependent/self-sufficient, undisciplined/controlled, relaxed/tense.

One of the factors much helpful in the prediction of behaviour is the internal or external focus of control. Another factor is the need for achievement (as opposite to the fear of success). Intelligent functioning of the individual depends on the speed of reactions, quick choice, ability to retrieve information, and the speed of reasoning. "Intelligence seems merely to set a minimum level below which entry into an occupation is difficult. Above this level, however, performance in a job is unrelated to intelligence» (p. 69). The organizational quality of work relationships seems to be much more important than intelligence of the employees or even of the manager.

Alexander J. MATEJKO

University of Alberta

La QVT une voie vers l'excellence, par Pierre R. Turcotte, Montréal, Les Éditions Agence d'Arc inc., 1988, 276 pp., ISBN 289022-134-2 et ISBN 2-7624-0066-x

Dans un monde fortement dominé par les préoccupations économiques, comme c'est le cas depuis la dernière récession, le concept d'excellence fait partie du discours quotidien au point que les gestionnaires sont incités à développer des axes d'excellence pour leur organisation. Les experts en management parlent de la nécessité d'une culture et d'une passion de l'excellence comme condition de réussite entrepreneuriale dans le contexte inexorable de la mondialisation des marchés et de l'internationalisation de la concurrence. L'ouvrage de Peters et Waterman (Le prix de l'excellence) reste le point d'encrage de cette tendance.

La réflexion que nous propose Pierre Turcotte sur l'amélioration des conditions de travail (amélioration du travail en soi et amélioration de l'environnement psychosocial) comme condition de réalisation de l'excellence organisationnelle, s'inscrit dans cette optique. Pour ce faire, il passe en revue les différents aspects du travail tels que l'organisation du travail, les conditions de travail, le climat organisationnel, la participation, la santé au travail et la productivité. En détail, l'ouvrage est réparti en sept chapitres dont voici un bref aperçu.

Dans le premier chapitre, l'auteur présente l'évolution des formes d'organisation du travail en expliquant les liens de causalité qui ont prévalu à leur apparition. Il s'agit, en quelque sorte, d'une introduction pour mieux comprendre le mouvement de QVT. Le second chapitre porte plus spécifiquement sur le courant de qualité de vie au travail, son origine, sa définition et les dimensions d'un programme de QVT. Le chapitre trois concerne la relation entre les facteurs organisationnels et la QVT. L'auteur précise que ces éléments peuvent aider l'organisation à réaliser ses objectifs de croissance, de profitabilité, de productivité, de qualité totale, etc. dans la mesure où ils sont imprégnés d'une qualité de vie au travail. Les deux chapitres subséquents étayent davantage le concept de QVT en mettant en relief l'importance de la participation et de la communication comme l'ossature et le coeur de la QVT. L'auteur analyse également la notion de la santé dans une perspective de développement organisationnel. Le sixième 
chapitre illustre une démarche d'implantation d'un programme de QVT et relate les stratégies, les difficultés et les implications d'un tel projet. Le dernier chapitre étudie la relation entre l'excellence et la QVT telle qu'expliquée dans les chapitres précédents. À ce titre, l'auteur croise chacun des huit attributs de l'excellence et les dimensions d'un programme de QVT.

En synthèse, le livre est constitué de deux pôles dont le premier, centré sur la notion de QVT, constitue près de $80 \%$ du volume (chap. 2, 3, 4, 5,6). Le second pôle, qui est l'objet du septième chapitre, établit la relation entre la QVT et l'excellence organisationnelle.

De façon concrète, l'ouvrage est une réflexion globale sur le concept de QVT. Ceci est d'autant plus intéressant que cette notion a souvent été présentée soit à travers des études de cas isolés faute d'une formulation synthèse ou soit de façon tellement théorique qu'elle n'est pas opérationnelle. L'originalité de Pierre Turcotte réside dans la mise en évidence des liens qui existent entre les huit attributs de l'excellence et les dimensions d'un programme de QVT (participation, communications, environnement de travail et design des tâches, voir tableau 7.2, p. 240). Cette relation est basée sur des considérations générales découlant d'une compréhension globale du concept de QVT qui préconise la réorganisation du travail de manière à favoriser la participation, le bien-être du travailleur et l'harmonisation du milieu de travail et de l'environnement hors travail. Par contre, en ce qui concerne la notion d'excellence, l'auteur se contente de faire simplement référence à la conception managériale de Peters et Waterman qui est un ensemble de principes. D'emblée, il devient relativement facile, voire évident, de conclure que toute organisation qui adopte une approche de QVT ne peut que tendre vers l'excellence, ce qui est vrai en principe, sauf que dans les faits, certaines questions restent sans réponse. Par exemple, on peut se demander pourquoi si peu d'entreprises, particulièrement les PME québécoises, adoptent cette approche, surtout quand elles peuvent espérer devenir excellentes. Pourquoi y a-t-il autant de programmes de QVT qui échouent ou sont arrêtés après quelques années? À notre avis, la dimension critique de la relation entre la QVT et l'excellence organisationnelle a été très peu mise en relief. Ce n'était peut-être pas la préoccupation immédiate de l'auteur qui, nous en sommes convaincu, est très certainement conscient des difficultés de réalisation d'un programme de QVT et des embûches qui parsèment le chemin de l'excellence.

En conclusion, l'ouvrage reste un outil d'éveil et de sensibilisation aux vertus de la QVT comme philosophie de gestion. En tant que tel, il peut certainement susciter l'émergence d'une culture de QVT comme un concept global lié à toute forme d'organisation et non pas comme un simple courant de pensée que certains considèrent déjà dépassé. Selon la perspective de ce livre, la ressource humaine reste la clef de survie des entreprises dans le contexte actuel de déréglementation de l'environnement économique et il est primordial de lui assurer une meilleure qualité de vie au travail.

Roger ZAORÉ

Université du Québec à Rimouski

Organizational Behavior, by Robert P. Vecchio, Chicago, Toronto, The Dryden Press, 1988, pp. 576, ISBN 0-000853-0

This textbook is a useful introduction to the research conducted on individual behavior in organizations. The first thirteen chapters present findings and conceptual schemes developed mainly by academic researchers in relation to topics of practical importance, such as learning, motivation, performance appraisal, leadership, group dynamics and stress. 